\title{
Better performance with performance budgeting? Analyzing cases of success and failure in public administrations.
}

Utz Helmuth

University of St. Gallen and Georgetown University - email: utz.helmuth@unisg.ch

\begin{abstract}
Research Problem
Performance Budgeting (PB) is associated with more freedom to manage and a focus on outcome orientation which should enhance the performance of public managers. However, evaluations have shown that results are rather diverse. It is the aim of this study to identify the underlying causal mechanisms to better understand success and failure of PB. To analyze the interplay of the different factors, this study uses a two-level theoretical concept with five hypotheses (figure 1).
\end{abstract}

\section{Data and Methods}

The study consists of 23 cases based on qualitative interviews with Swiss public managers from the municipal or canton (regional) level and some subsequent document analyses. It focuses on the perceptions of these managers.

The study combines a cross-case design of typological theory with methods of within-case analysis. Typology theory defines independent variables distinguished in different categories, for which multiple cases and their outcomes can be linked. The data-analysis of this study uses the codes $\mathrm{Y}, \mathrm{N}, \mathrm{Y} / \mathrm{N}$ with ordinal scale and focuses on six variables (table 2). The within-case analysis is to investigate and explain the decision process that leads to these deviant outcomes and is influenced by techniques of process tracing.

\section{Results}

Results question the claim of PB to be a universal management concept. Type of department has a strong influence on the degree of freedom perceived by public managers. Parliamentary behavior has some moderate impact on perceived freedom. Degree of perceived freedom has the highest influence on success of PB. Project management seems to have a minor influence, though data base of this study is small in this respect. The influence of a prior career in the private sector for performance is marginal (figure 2).

Table 1: Typological table of performance budgeting

\begin{tabular}{|c|c|c|c|c|c|c|c|}
\hline Set & Cases & $\begin{array}{l}\text { IV: Tasks of } \\
\text { department }\end{array}$ & \begin{tabular}{|l} 
IV: \\
Parliament \\
behaves \\
according to \\
concept \\
\end{tabular} & \begin{tabular}{|l} 
IMV: \\
Freedom \\
perceived?
\end{tabular} & \begin{tabular}{|l} 
IV: \\
Introduction \\
\& Project \\
Mgmt OK
\end{tabular} & $\begin{array}{l}\text { IV: Prior } \\
\text { career in the } \\
\text { private } \\
\text { sector? }\end{array}$ & $\begin{array}{l}\text { DV: } \\
\text { Outcome: } \\
\text { Did it work? }\end{array}$ \\
\hline 1 & $\begin{array}{l}E_{1} \\
D_{1} \\
A_{1} \\
C_{1}\end{array}$ & Business-like & $Y$ & $Y$ & $Y$ & Y & $Y$ \\
\hline 2 & $A_{2}$ & Business-like & $Y$ & $Y$ & $\mathrm{Y} / \mathrm{N}$ & $Y$ & $Y$ \\
\hline 3 & $\begin{array}{l}\mathrm{B}_{1} \\
\mathrm{~B}_{2}\end{array}$ & Business-like & $\mathrm{Y} / \mathrm{N}$ & Y & $Y$ & $Y$ & $Y$ \\
\hline 4 & $E_{2}$ & Business-like & $Y$ & $Y$ & $Y$ & $\mathrm{~N}$ & $Y$ \\
\hline 5 & $A_{3}$ & Business-like & $\mathrm{N}$ & $\mathrm{N}$ & $Y$ & $Y$ & $\mathrm{Y} / \mathrm{N}$ \\
\hline & & & & & & & \\
\hline 6 & $E_{3}$ & Educ/Sports & $Y$ & $Y$ & $Y$ & $Y$ & $Y$ \\
\hline 7 & $c_{2}$ & Educ/Sports & $Y$ & $Y$ & $Y$ & $\mathrm{~N}$ & $Y$ \\
\hline 8 & $B_{3}$ & Educ/Sports & $Y$ & $\mathrm{~N}$ & $Y$ & $\mathrm{~N}$ & $\mathrm{~N}$ \\
\hline 9 & $D_{2}$ & Educ/Sports & $\mathrm{Y} / \mathrm{N}$ & $Y$ & $Y$ & $\mathrm{~N}$ & $Y$ \\
\hline 10 & $E_{4}$ & Educ/Sports & $\mathrm{Y} / \mathrm{N}$ & $Y$ & $Y$ & $Y$ & $Y$ \\
\hline 11 & & Internal & & & $y$ & $N$ & $\mathrm{Y} / \mathrm{N}$ \\
\hline \begin{tabular}{|l|}
11 \\
12
\end{tabular} & \begin{tabular}{|l}
$\mathrm{A}_{4}$ \\
$\mathrm{D}_{3}$
\end{tabular} & \begin{tabular}{|l|} 
Internal \\
Internal
\end{tabular} & $\frac{\mathrm{N}}{\mathrm{N}}$ & $\frac{N}{Y / N}$ & $\frac{\mathrm{Y}}{\mathrm{Y} / \mathrm{N}}$ & $\frac{N}{Y}$ & $\frac{Y / N}{Y / N}$ \\
\hline 13 & $D_{4}$ & Internal & $Y$ & $\mathrm{Y} / \mathrm{N}$ & $\mathrm{Y} / \mathrm{N}$ & $\mathrm{N}$ & $\mathrm{Y} / \mathrm{N}$ \\
\hline \begin{tabular}{|l|}
14 \\
\end{tabular} & $E_{5}$ & Internal & $\mathrm{Y} / \mathrm{N}$ & $\mathrm{Y} / \mathrm{N}$ & $Y$ & $\mathrm{~N}$ & $Y$ \\
\hline 15 & $D_{5}$ & Internal & $\mathrm{Y} / \mathrm{N}$ & $\mathrm{Y} / \mathrm{N}$ & $Y$ & $Y$ & $Y$ \\
\hline 16 & $D_{6}$ & Internal & $\mathrm{Y}$ & $Y$ & $Y$ & $\mathrm{~N}$ & $Y$ \\
\hline & & & & & & & \\
\hline 17 & $B_{4}$ & Social & $Y$ & $\mathrm{Y} / \mathrm{N}$ & $Y$ & $Y$ & $\mathrm{Y} / \mathrm{N}$ \\
\hline 18 & $A_{5}$ & Social & $Y$ & $\mathrm{~N}$ & $Y$ & $Y$ & $\mathrm{~N}$ \\
\hline 19 & $c_{3}$ & Social & $\mathrm{N}$ & $\mathrm{Y} / \mathrm{N}$ & $Y$ & $\mathrm{~N}$ & $\mathrm{Y} / \mathrm{N}$ \\
\hline
\end{tabular}
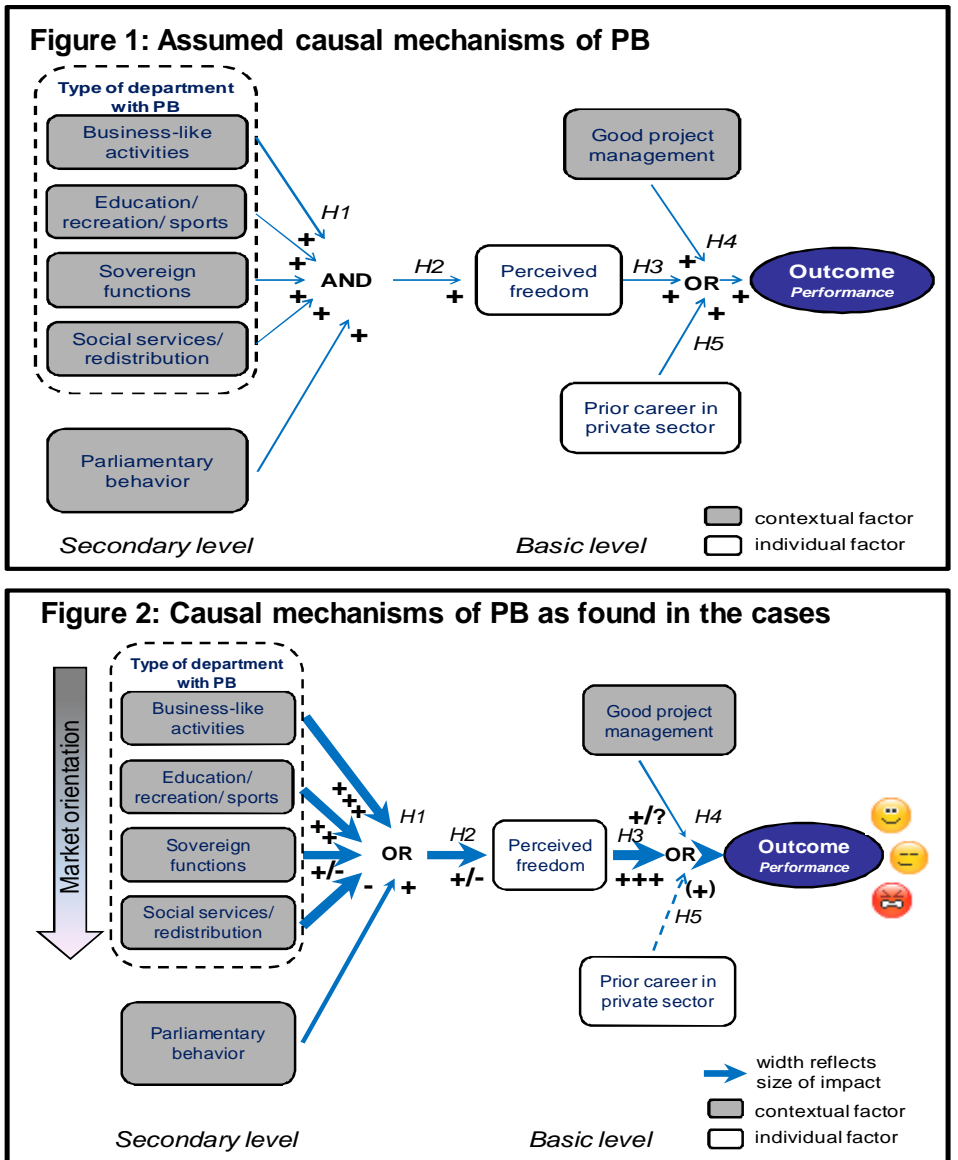

\section{Conclusion \& Implications}

- Perceived freedom is the dominating variable; goes along with the literature $=>$ should be considered when applying PB.

- Other than assumed, nature of task strongly influences perceived freedom => results question application of PB in nonmarket oriented departments.

- To some extent parliamentary behavior can constrain perceived freedom => parliaments need play according to the rules. 